\title{
Impact of Bacterial and Viral Challenge on Multidrug Resistance in First- and Third-Trimester Human Placenta
}

\author{
Phetcharawan Lye, ${ }^{*}$ Enrrico Bloise, ${ }^{* \dagger}$ Mohsen Javam, ${ }^{*}$ William Gibb, ${ }^{\ddagger \S}$ Stephen J. Lye, ${ }^{* \mathbb{\|} \| * *}$ and Stephen G. Matthews ${ }^{* \mathbb{\Psi} \| * *}$
}

From the Departments of Physiology, * Obstetrics and Gynecology, " and Medicine, "University of Toronto, Toronto, Ontario, Canada; the Laboratory of Translational Endocrinology, ${ }^{\dagger}$ Carlos Chagas Filho Biophysics Institute, Federal University of Rio de Janeiro, Rio de Janeiro, Brazil; the Departments of Cellular and Molecular Medicine $e^{\ddagger}$ and Obstetrics and Gynecology, ${ }^{\S}$ University of Ottawa, Ottawa, Ontario, Canada; and the Lunenfeld-Tanenbaum Research Institute, ** Mount Sinai Hospital, Toronto, Ontario, Canada

Accepted for publication

February 3, 2015.

Address correspondence to Stephen G. Matthews, Ph.D., Department of Physiology, Faculty of Medicine, University of Toronto, Medical Sciences Bldg., 1 King's College Circle, Toronto, ON M5S 1A8, Canada. E-mail: stephen.matthews@ utoronto.ca.

\begin{abstract}
The $\mathrm{ABC}$ transporters $\mathrm{P}$-glycoprotein (P-gp, official gene symbol $A B C B 1$ ) and breast cancer resistance protein (BCRP, official gene symbol $A B C G 2$ ) protect the conceptus from exposure to toxins and xenobiotics present in the maternal circulation. Viral or bacterial challenges alter expression of placental multidrug transporters in rodents. We hypothesized that exposure to lipopolysaccharide (LPS, bacterial antigen) and polyinosinicpolycytidylic acid (poly(I:C), viral antigen) would decrease P-gp and BCRP in the human placenta. Placental explants from first and third trimesters were challenged with 0.1 to $10 \mu \mathrm{g} / \mathrm{mL}$ LPS or 1 to $50 \mu \mathrm{g} / \mathrm{mL}$ poly(I:C) for 4 or 24 hours; mRNA levels, protein expression, and localization were assessed by quantitative real-time PCR, Western blot analysis, and immunohistochemistry, respectively. Toll-like receptor (TLR)-3 and TLR-4 mRNA expression increased from the first to third trimester $(P<0.01)$, and the receptors localized to cytotrophoblasts in the first trimester and to syncytiotrophoblasts in the third trimester. LPS exposure in first-trimester explants decreased $(P<0.001)$ ABCB1 and ABCG2 mRNA and protein levels. In contrast, poly $(\mathrm{I}: \mathrm{C})$ decreased $(P<0.05)$ ABCB1, TLR-3, and TLR-4 mRNA levels in the third trimester but not first trimester. LPS and poly(I:C) treatments increased $(P<0.01)$ IL-8 and chemokine ligand 2. Results suggest that bacterial infections likely alter exposure of the conceptus to toxins and drugs during early pregnancy, whereas viral infections may disrupt fetal protection in later stages of pregnancy. (Am J Pathol 2015, 185: 1666-1675; http://dx.doi.org/10.1016/j.ajpath.2015.02.013)
\end{abstract}

The placenta supports the growth and development of the fetus through hormone production and by enabling the transport of oxygen and nutrients from mother to fetus. It also plays an important role in protecting the fetus from substances in the maternal blood that would otherwise be detrimental to the conceptus and its development, such as glucocorticoids and environmental toxins (eg, organophosphate pesticides and endocrine disruptors). ${ }^{1-3}$ This barrier function is supported by a series of proteins within the trophoblast, including membranebound transporters (multidrug resistance proteins) that efflux unwanted factors that enter the trophoblast, back into the maternal circulation.

Two multidrug transporter proteins, P-glycoprotein (P-gp; encoded by the $A B C B 1$ gene) and the breast cancer resistance protein (BCRP; encoded by the $A B C G 2$ gene), are enriched at the apical membrane of syncytiotrophoblast layer ${ }^{3-6}$ and play an important role in protecting the fetus from exposure to steroids, environmental toxins, and xenobiotics. ${ }^{3,7}$ We have reported that placental P-gp expression decreases progressively with advancing gestation, whereas placental BCRP levels increase toward term, ${ }^{4-6}$ demonstrating a gestational age-dependent pattern of expression. Expression of these proteins is regulated by a number of factors, including placental hormones $^{8-10}$ and oxygen tension. In the case of oxygen, the effect on P-gp and BCRP expressions is gestational age dependent. ${ }^{4,11}$

Supported by the Canadian Institutes for Health Research grant FRN-57746 (S.G.M. and W.G.).

Disclosures: None declared. 
Table 1 List of Primers Used in This Study

\begin{tabular}{|c|c|c|}
\hline Primer name & Sequence & Reference \\
\hline $\mathrm{ABCB} 1^{*}$ & Forward: 5'-AGCAGAGGCCGCTGTTCGTT-3' & \\
\hline \multirow[t]{2}{*}{$\mathrm{ABCG} 2^{*}$} & Forward: 5'-TGGAATCCAGAACAGAGCTGGGGT-3' & \\
\hline & Reverse: 5'-AGAGTTCCACGGCTGAAACACTGC-3' & \\
\hline \multirow[t]{2}{*}{ TLR-3 $3^{\dagger}$} & Forward: 5'-TTACGAAGAGGCTGGAATGG-3' & \\
\hline & Reverse: 5'-AGGAACTCCTTTGCCTTGGT-3' & \\
\hline \multirow[t]{2}{*}{$\mathrm{CCL}^{*}$} & Forward: 5'-TTCATTCCCCAAGGGCTCGCTCA-3' & \\
\hline & Reverse: 5'-AGCACAGATCTCCTTGGCCACAA-3' & \\
\hline \multirow[t]{2}{*}{ IL-6 } & Forward: 5'-TTGTCAAGACATGCCAAAGTGCT-3' & 24 \\
\hline & Reverse: 5'-GCCTCAGACATCTCCAGTCC-3' & \\
\hline $\mathrm{IL}-8^{*}$ & Forward: 5'-GCAGCCTTCCTGATTTCTGCAGCT-3' & \\
\hline YWHAZ & Reverse: 5'-CCGCCAGGACAAACCAGTAT-3' & \\
\hline \multirow[t]{2}{*}{ CYC1 } & Forward: 5'-CAGATAGCCAAGGATGTGTG-3' & 25 \\
\hline & Reverse: 5'-CATCATCAACATCTTGAGCC-3' & \\
\hline \multirow[t]{2}{*}{ TOP1 } & Forward: 5'-GATGAACCTGAAGATGATGGC-3' & 25 \\
\hline & Reverse: 5'-TCAGCATСАТССТСАТСТCG-3' & \\
\hline
\end{tabular}

${ }^{*}$ Gene specific primers were designed with Primer-BLAST (http://www.ncbi.nlm.nih.gov/tools/primer-blast).

${ }^{\dagger}$ Program primers 3-In silico PCR.

Evidence also indicates that the expression and function of these transporters may be disrupted by infection and inflammation. ${ }^{3,12}$ This is important because infection and inflammation during pregnancy are associated with complications of pregnancy such as preterm birth and fetal brain damage. ${ }^{13}$ In rodents, bacterial exposure and proinflammatory cytokines alter Abcbla mRNA expression and function in the placenta and other tissues. $^{12,14,15}$ Viral infection decreases expression of Abcb1a, Abcb1b, and Abcg2 in the rat placenta but increases expression in the liver. ${ }^{16}$ These data suggest that infective agents alter multidrug resistance in a tissue- and infective agentdependent manner and highlight the need for further studies to investigate how inflammatory mediators, through their actions on the multidrug resistance proteins, might expose the conceptus to potential harmful substances in the maternal circulation.

To date no studies have examined the effect of lipopolysaccharide (LPS; modeling bacterial infection) or polyinosinicpolycytidylic acid (poly(I:C); modeling viral infection) on P-gp and/or BCRP in the human placenta in early and late gestation. However, their respective receptors, Toll-like receptor (TLR)-4 and TLR-3, are expressed in the placenta at term and preterm. ${ }^{17-19}$ Therefore, our aim was to examine the effect of LPS and poly(I:C) on placental P-gp and BCRP expressions in the first and third trimesters of human pregnancy by using placental villous cultures, which maintain tissue integrity during culture. We hypothesized that bacterial or viral infection would reduce placental expression of the multidrug transporters P-gp and BCRP in a gestational age-dependent fashion.

\section{Materials and Methods}

\section{Placental Tissue Collection and Ethical Approval}

Placental specimens were collected by the Research Centre for Women's and Infants' Health BioBank program of the Mount Sinai Hospital after informed consent and in adherence with the policies of Mount Sinai Hospital and the University of Toronto Research Ethic Boards. First-trimester tissues were obtained at 8 to 10 weeks' gestation from patients undergoing surgical termination of pregnancy and at $>37$ weeks' gestation from term elective cesarean deliveries.

\section{Placental Villous Explants}

Placental villous explants were cultured as described previously, ${ }^{4,20,21}$ with minor alterations. Briefly, placental specimens were placed into $1 \%$ phosphate-buffered saline (PBS) with $\mathrm{Ca}^{2+}$ and $\mathrm{Mg}^{2+}$ and transported to the laboratory. Tissues were dissected into villous clusters of approximately 15 to $30 \mathrm{mg}$, and three villous explants were cultured per well in 12-well plates that contained Dulbecco's modified Eagle's medium/F12, Normocin antibiotic (Invivogen, San Diego, CA), and $1 \times$ insulin, transferrin, and selenium-A (Invitrogen, Grand Island, NY) that was previously equilibrated at $8 \% \mathrm{O}_{2}\left(\mathrm{CO}_{2}, 37^{\circ} \mathrm{C}\right)$ for 24 hours. Explants were cultured for 24 hours and then randomly divided into treatment groups. Explants were treated with the TLR-4 ligand, LPS from Escherichia coli (0.1, 1, 10 $\mu \mathrm{g} / \mathrm{mL}^{21-23}$; Sigma-Aldrich, St. Louis, MO), or the TLR-3 

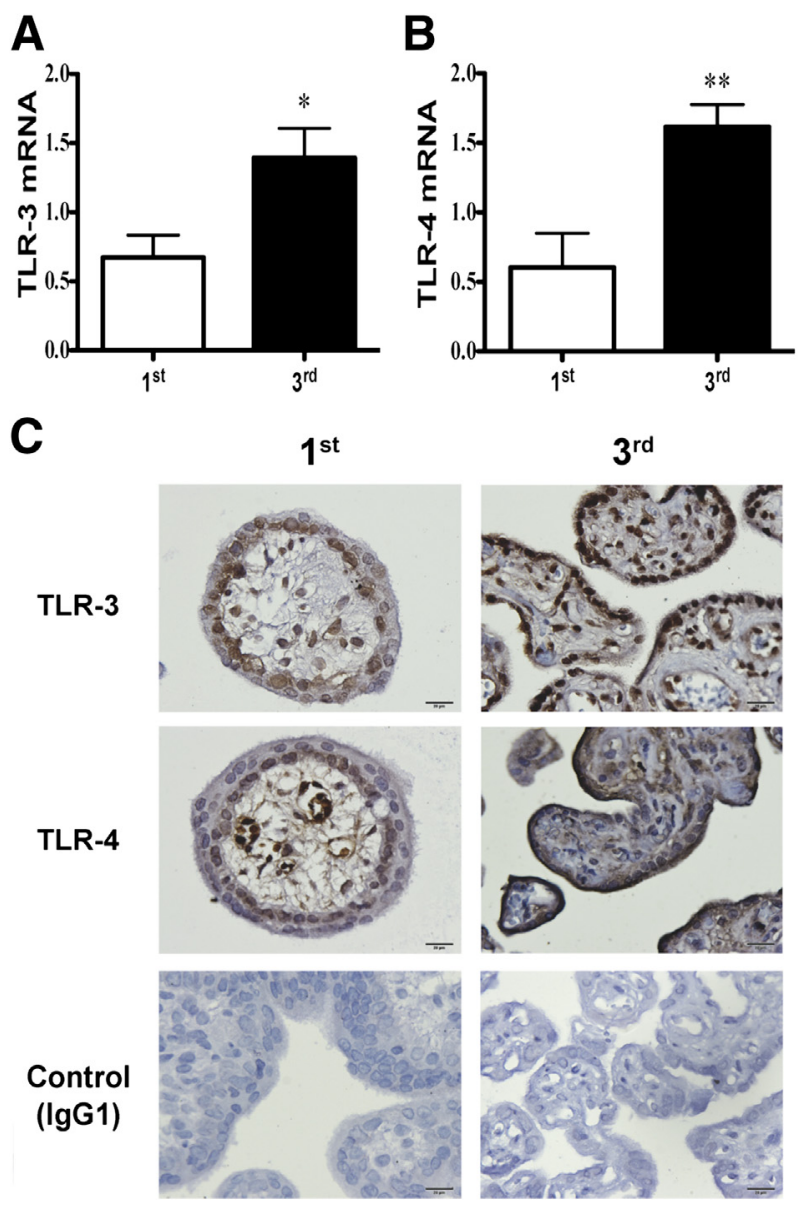

Figure 1 Expression and localization of TLR-3 and TLR-4 in the firstand third-trimester placental villous explants. TLR-3 (A) and TLR-4 (B) mRNA levels. C: Localization of TLR-3 and TLR-4 protein in the first- and third-trimester human placenta. TLR-3 and TLR- 4 protein are expressed in the cytotrophoblast in the first trimester and in the syncytiotrophoblast in the third trimester. TLR-3 is also present in the villous core of thirdtrimester placental explants. Data are expressed as means \pm SEM. $n=4$ per group (C). Statistical analysis was conducted with an unpaired $t$-test. ${ }^{*} P<0.05,{ }^{* *} P<0.01$. TLR, Toll-like receptor.

ligand poly(I:C) $\left(1,10,50 \mu \mathrm{g} / \mathrm{mL}^{16}\right.$; Sigma-Aldrich) for either 4 or 24 hours. Explants were then collected and stored at $-80^{\circ} \mathrm{C}$ for total RNA and protein extraction or were fixed in $4 \%$ paraformaldehyde for histology and immunohistochemistry. The culture media was collected to measure lactate dehydrogenase (LDH) and to assess tissues viability during culture (Roche Applied Science, Indianapolis, IN) as previously described. ${ }^{4,11}$

\section{Quantitative Real-Time PCR}

Total RNA was isolated from explants by using RNeasy Plus Universal Mini Kit (Qiagen, Toronto, ON, Canada) according to the manufacturer's instructions. RNA concentration and purity were assessed with the NanoDrop 1000 Spectrophotometer (Thermo Scientific, Wilmington, DE) and Experion RNA StdSens Analysis Kit (Bio-Rad, Mississauga, ON, Canada), respectively. RNA was reverse-transcribed to cDNA by using the iScript Reverse Transcription Supermix (Bio-Rad). mRNA levels of the $A B C B 1$ (P-gp), ABCG2 (BCRP), TLR3, TLR4, CXCL8 (IL8) (a proinflammatory cytokine), and chemokine (C-C motif) ligand 2 (CCL2) genes were measured by quantitative real-time PCR by using SYBR Green reagent (Sigma-Aldrich) and the CFX 380 Real-Time system C 1000 TM Thermal Cycle (Bio-Rad), with the following cycling conditions: initial enzyme $95^{\circ} \mathrm{C}$ for 5 minutes followed by 38 cycles of $95^{\circ} \mathrm{C}$ for 15 seconds and $60^{\circ} \mathrm{C}$ for 20 seconds. Gene-specific primers are shown in Table $1 .{ }^{24,25}$ Gene expression was normalized to the geometric mean of the following selected reference genes (Table 1) that showed stable expression levels after LPS and poly(I:C) treatments in first- and third-trimester tissues and the zeta polypeptide (YWHAZ) and succinate-ubiquinone oxidoreductase (SDHA) genes in the first trimester; YWHAZ and DNA topoisomerase 1 (TOPI) in the third trimester. Levels of placental TLR-3 and TLR-4 mRNA from first- and third-trimester explants were normalized to the geometric mean of YWHAZ and cytochromec-1 (CYC1), which showed stable expression levels in the first- and third-trimester tissues.

\section{Immunohistochemistry}

TLR-3, TLR-4, and the neurotrophic tyrosine kinase receptor type 2 (NTRK2) were localized in tissues before and after culture with LPS and poly(I:C). Slides were dewaxed in xylene and rehydrated in ethanol in descending gradients. Endogenous peroxidase activity was blocked with $0.3 \% \mathrm{H}_{2} \mathrm{O}_{2}$ in methanol for 30 minutes and washed 10 minutes in PBS. Antigen retrieval was performed by preheating sections in $10 \mathrm{mmol} / \mathrm{L}$ sodium citrate. Sections were again washed in PBS before blocking in protein blocking solution (Dako, Burloak, ON, Canada) for 1 hour. Slides were incubated overnight with the following primary antibodies: TLR-3 (dilution 1:100; Abcam, Toronto, ON, Canada), TLR-4 (dilution 1:200; Abcam), and NTRK2 (dilution 1:500; Abcam). Mouse or rabbit IgG1 were added instead of primary antibody in controls. Slides were re-washed in PBS and incubated with the following secondary antibodies: goat anti-mouse (TLR-3/-4) and goat anti-rabbit (NTRK2) (dilution 1:300; Dako). Sections were washed in PBS and incubated with streptavidin-horseradish peroxidase (HRP) for 1 hour (Dako); staining was detected with the peroxidase substrate kit DAB (Dako). Slides were counterstained with hematoxylin, dehydrated in ascending gradients of ethanol, and coverslipped. Slides were visualized with an Olympus BX61 upright, motorized microscope with an Olympus DP72 digital camera (Olympus, Tokyo, Japan).

\section{LDH Cytotoxic Assay}

Viability of the explants subjected to treatment with LPS $(0.1,1,10 \mu \mathrm{g} / \mathrm{mL})$ or poly(I:C) $(1,10,50 \mu \mathrm{g} / \mathrm{mL})$ was determined by measuring LDH leakage into the medium as previously described. ${ }^{4,11}$ Briefly, LDH was quantified with 
A

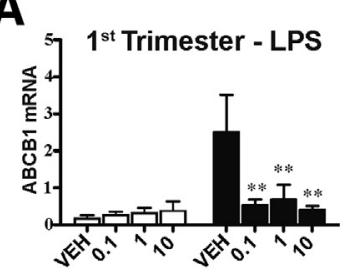

B
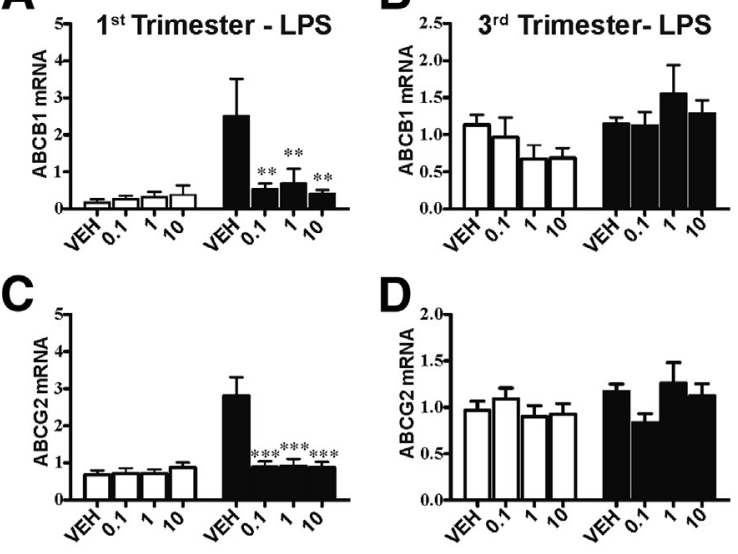

\section{D}

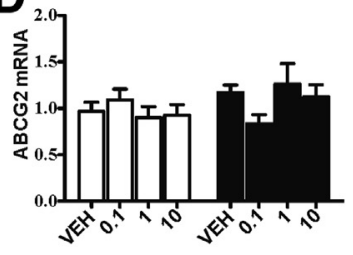

$\mathbf{F}$

E

VEH-4h 10-4h VEH-24h 10-24h VEH-4h 10-4h VEH-24h 10-24h

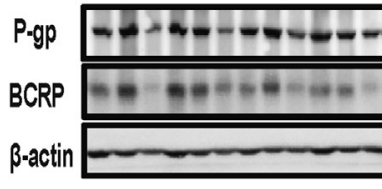

G $1^{\text {st }}$ Trimester - LPS
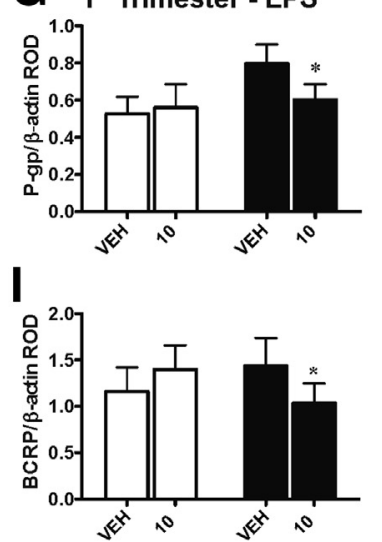

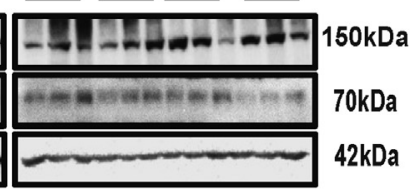

H $3^{\text {rd }}$ Trimester- LPS

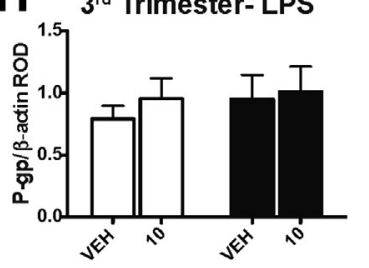

$\mathbf{J}$

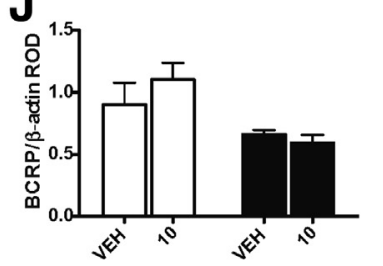

Figure 2 Effect of LPS on ABCB1 (P-gp) and ABCG2 (BCRP) mRNA and protein expression in the first- and third-trimester placental villous explants. $A B C B 1$ (A) and ABCG2 (C) mRNA levels in placental explants (8 to 10 weeks) and $A B C B 1$ (B) and ABCG2 (D) mRNA levels in placental explants (38 to 40 weeks, cesarean delivery) treated with 0.1 to $10 \mu \mathrm{g} / \mathrm{mL} \mathrm{LPS} \mathrm{for} 4$ (white bars) or 24 (black bars) hours. LPS reduces ABCB1 and ABCG2 mRNA levels in the first- but not the third-trimester placental explants. Representative Western blot analysis of P-gp and BCRP levels in the first $(\mathbf{E})$ and third $(\mathbf{F})$ trimester placenta. Placental explants were treated with $10 \mu \mathrm{g} / \mathrm{mL}$ LPS for 4 or 24 hours or with VEH. Densitometric analysis of P-gp (G and $\mathbf{H})$ and BCRP ( $\mathbf{I}$ and $\mathbf{J}$ ) in the first trimester and third trimester, respectively, normalized to $\beta$-actin. LPS decreases P-gp and BCRP levels in the first trimester but has no effect on P-gp or BCRP levels in the third-trimester placental explants. Data are expressed as means \pm SEM. $n=6$ per group. Statistical differences were tested by two-way analysis of variance, followed by Bonferroni's post test. ${ }^{*} P<0.05,{ }^{* *} P<0.01$, and ${ }^{* * *} P<0.001$ versus VEH. BCRP, breast cancer resistance protein; LPS, lipopolysaccharide; P-gp, P-glycoprotein; ROD, relative optical density; VEH, vehicle.

the Cytotoxicity Detection kit (Roche Applied Science) according to the manufacturer's instructions. A standard curve for the LDH assay was generated with L-LDH from rabbit muscle (Sigma-Aldrich), whereas absorbance was measured at $490 \mathrm{~nm}$ (BioTek Instrument Inc., Winooski,

VT). The LDH concentration in the media was normalized to placental explant weight.

\section{Western Blot Analysis}

Western blot analysis was conducted as previously described. ${ }^{5}$ Briefly, protein from placental tissues and explants was extracted with sonication by using lysis buffer $(1 \mathrm{~mol} / \mathrm{L}$ TrisHCL pH 6.8, 2\% SDS, $10 \%$ glycerol that contained protease and phosphatase inhibitor cocktail; Thermo Scientific). Protein concentration was determined with Thermo Scientific Pierce BCA Protein Assay kit (Thermo Scientific). Proteins were separated by electrophoresis (100 V, 1 hour) by using $7 \%$ SDSpolyacrylamide (P-gp and BCRP loaded with $30 \mu \mathrm{g}$ per well). Protein was then transferred to polyvinylidene fluoride membrane by using Transfer Pack Quick Start Guide (Bio-Rad) for 10 minutes. Membranes were blocked with $5 \%$ bovine serum albumin in tris-buffered saline with Tween (1 hour) for all proteins except anti- $\beta$-actin, where $5 \%$ skim milk was used. Primary antibodies used for the analyses were anti-rabbit MDR1 (dilution 1:1000; Abcam), anti-mouse BCRP (dilution 1:500; BXP-21; Santa Cruz Biotechnology, Dallas, TX), and anti-goat $\beta$-actin (Santa Cruz Biotechnology). Blots were incubated with primary antibodies overnight at $4{ }^{\circ} \mathrm{C}$ in $5 \%$ bovine serum albumin or skim milk in tris-buffered saline with Tween as described above. The polyvinylidene fluoride membranes were subsequently incubated for 1 hour with HRP-linked anti-mouse secondary antibody (GE Healthcare Bio-Science, Baie d'Urfe, QC, Canada) at concentrations of 1:10,000 for P-gp or 1:15,000 for BCRP. Anti-goat secondary antibody (Bio-Rad) linked to HRP (dilution 1:10,000) was used for $\beta$-actin. Protein-antibody complexes were detected by incubating with Laminate Crescendo Western HRP Substrate (Millipore, Oak Drive, CA) for 3 minutes, and the chemiluminescence was detected under UV by using Versa Doc system (Bio-Rad). The protein band intensity was quantified with ImageJ 1.46r (NIH, Bethesda, MD).

\section{Statistical Analysis}

Exploratory data analyses were performed with Prism version 6 (GraphPad Software Inc., San Diego, CA). Differences in mRNA levels between first and third trimesters were assessed with unpaired $t$-tests. Differences in mRNA levels in explants exposed to LPS and poly(I:C) were assessed with two-way analysis of variance, followed by Bonferroni's test. Differences in cytokine and protein levels were assessed with a paired $t$-test. Differences were considered statistically significant when $P<0.05$.

\section{Results}

TLR-3 and TLR-4 Expression and Localization in the First- and Third-Trimester Placenta

Both first- and third-trimester placental explants expressed TLR-3 and TLR-4 mRNA. Levels of both receptors were 
A

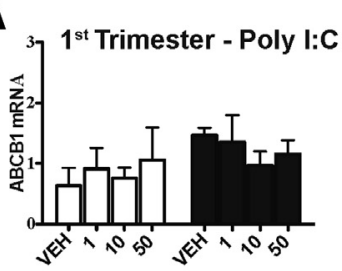

B

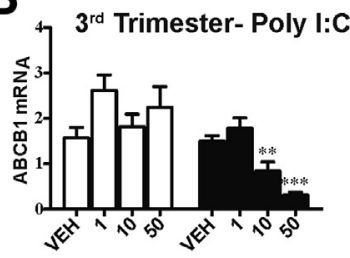

C

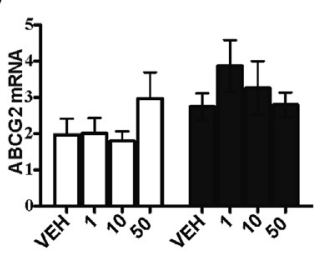

D

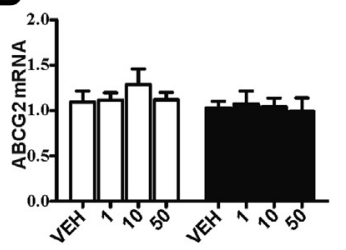

E
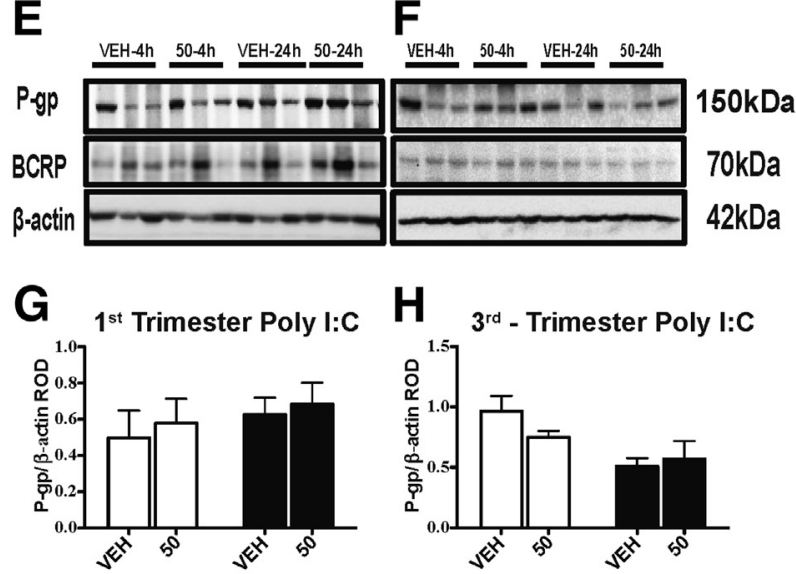

H $3^{\text {rd }}$ - Trimester Poly I:C

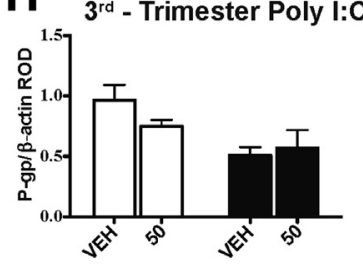

I

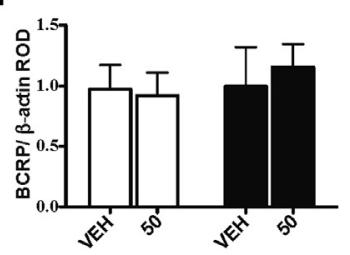

J

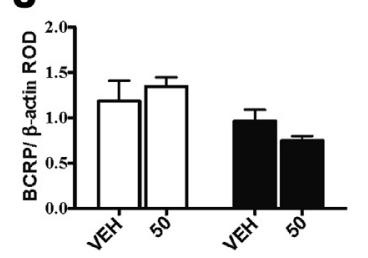

Figure 3 Effect of poly(I:C) on ABCB1 (P-gp) and ABCG2 (BCRP) mRNA and protein expressions in the first- and third-trimester placental villous explants. ABCB1 (A) and ABCG2 (C) mRNA levels of placental explants (8 to 10 weeks) and ABCB1 (B) and ABCG2 (D) mRNA levels of placental explants (38 to 40 weeks, normal term cesarean delivery) treated with 1 to $50 \mu \mathrm{g} / \mathrm{mL}$ poly(I:C) for 4 (white bars) or 24 (black bars) hours. No effect of poly(I:C) was found on mRNA levels of any of the genes in the first trimester; however, poly(I:C) decreases $A B C B 1$ mRNA levels after 24 hours of exposure. Representative Western blot analysis of P-gp and BCRP protein levels in the first (E) and third (F) trimester placenta. Placental explants were treated with $50 \mu \mathrm{g} / \mathrm{mL}$ poly(I:C) for 4 or 24 hours or with VEH. Densitometric analysis of P-gp $(\mathbf{G}$ and $\mathbf{H})$ and BCRP ( $\mathbf{I}$ and $\mathbf{J}$ ) in first trimester and third trimester, respectively, normalized to $\beta$ actin. No effect of poly(I:C) was found on BCRP or P-gp proteins in the first- and third-trimester placental explants. Data are expressed as means \pm SEM. $n=6$ per group. Statistical differences were tested by two-way analysis of variance, followed by Bonferroni's post test. ${ }^{*} P<0.01,{ }^{* * *} P<0.001$ versus vehicle. BCRP, breast cancer resistance protein; P-gp, P-glycoprotein; poly(I:C), polyinosinic-polycytidylic acid; ROD, relative optical density; VEH, vehicle.

higher in the third trimester than in the first trimester $(P<0.05$ and $P<0.01$, respectively) (Figure 1, A and B). Accordingly, TLR-3 and TLR-4 immunostaining intensity was increased in third-trimester explants. In the first

trimester, TLR-3 and TLR-4 protein localized to the cytotrophoblast, whereas in the third trimester both localized to the syncytiotrophoblast. In addition, third-trimester explants also exhibited TLR-3 immunostaining in cells of the villous core (Figure 1C).

\section{Effect of LPS on Expression of ABCB1 and ABCG2 mRNA and Protein Expression}

To simulate the effects of bacterial infection on P-gp and BCRP expression, villous explants were treated with three doses of LPS $(0.1,1,10 \mu \mathrm{g} / \mathrm{mL})$ for 4 and 24 hours. A gestational age-dependent effect of LPS was found on $\mathrm{ABCB} 1$ and ABCG2 mRNA and protein levels. Although a significant decrease was observed in $\mathrm{ABCB} 1(P<0.01)$ and ABCG2 mRNA $(P<0.001)$ levels at 24 hours after LPS treatment in the first-trimester explants (Figure 2, A and C), no effect was observed on ABCB1 and ABCG2 mRNA levels in third-trimester explants (Figure 2, B and D). Similarly, LPS decreased P-gp and BCRP protein levels (Figure 2, E and F) $(P<0.05)$ after 24 hours in first-trimester explants (Figure 2, G and I) but did not alter P-gp and BCRP levels in the thirdtrimester explants (Figure 2, $\mathrm{H}$ and $\mathbf{J}$ ).

Effect of Poly(I:C) on Expression of $A B C B 1$ and $A B C G 2$ mRNA and Protein Expression

To simulate the effects of viral infection on P-gp and BCRP expressions, villous explants were treated with three doses of poly(I:C) $(1,10,50 \mu \mathrm{g} / \mathrm{mL})$ for 4 and 24 hours. Poly(I:C) did not alter $\mathrm{ABCB} 1$ or $\mathrm{ABCG} 2 \mathrm{mRNA}$ or protein levels in the first-trimester explants (Figure 3, A, C, E, G, and I). In third-trimester explants, poly(I:C) induced a dose-dependent decrease in ABCB1 mRNA levels after 24 hours $(P<0.05)$ (Figure 3B). However, poly(I:C) had no effect on ABCG2 mRNA levels or the protein levels of either transporter (Figure 3, D, F, H, and J).

\section{Effect of LPS and Poly(I:C) on TLR-3 and TLR-4 mRNA Expression}

Two-way analysis of variance analysis revealed a significant increase in TLR-3 and TLR-4 mRNA levels over the 24-hour culture period in the first-trimester explants. However, LPS treatment did not alter TLR-3 or TLR-4 mRNA levels in either the first- or third-trimester tissues (Figure 4, A-D). Poly(I:C) had no effect on TLR-3 or TLR-4 expression in the first-trimester explants but induced a dose-dependent decrease $(P<0.001)$ of TLR-3 and TLR- 4 mRNA levels in the third-trimester explants (Figure 4, E-H).

\section{Placental Inflammatory Response to LPS and Poly(I:C)}

To confirm that the explants were responsive to LPS and poly(I:C) the expression of the proinflammatory markers IL8 and the chemokine CCL2 was evaluated. In the first 
A

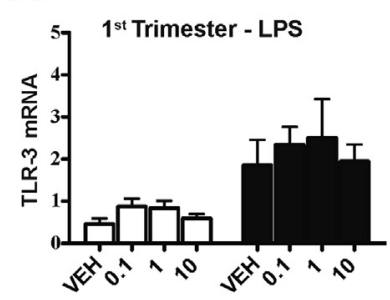

B

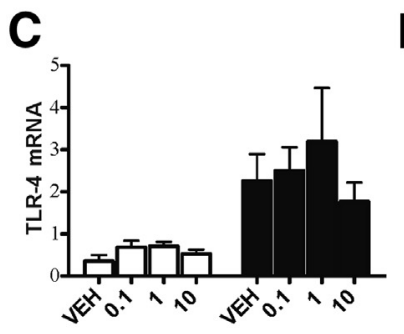

E
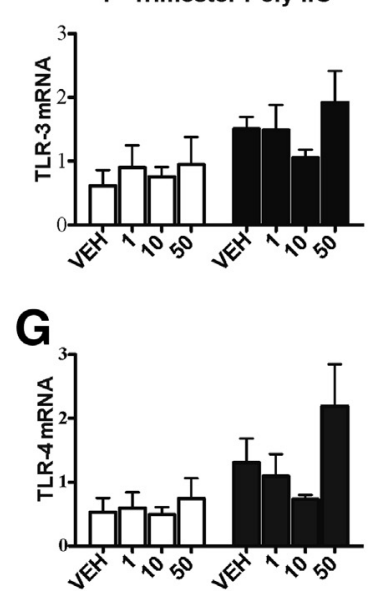

$\mathbf{H}$

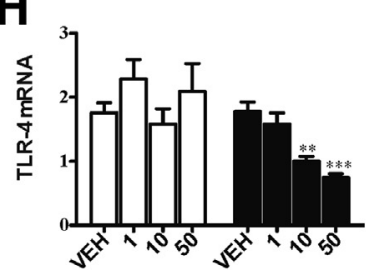

Figure 4 Effect of LPS and poly(I:C) on expression of TLR-3 and TLR-4 mRNA in the first- and third-trimester placental villous explants. TLR-3 (A) and TLR-4 (C) mRNA expression of placental explants (8 to 10 weeks) and TLR-3 (B) and TLR-4 (D) mRNA levels (38 to 40 weeks, normal term cesarean delivery) of placental villous explants treated with 0.1 to $10 \mu \mathrm{g} / \mathrm{mL}$ LPS for 4 (white bars) or 24 (black bars) hours. TLR-3 (E) and TLR-4 (G) mRNA levels ( $n=6$ per group) of placental explants ( 8 to 10 weeks) and TLR-3 (F) and TLR-4 (H) mRNA levels (38 to 40 weeks, normal term cesarean delivery) of placental explants treated with 1 to $50 \mu \mathrm{g} / \mathrm{mL}$ poly(I:C) for 4 or 24 hours. Data are expressed as means \pm SEM. $n=6$ per group. Statistical differences were tested by two-way analysis of variance, followed by Bonferroni's post test. ${ }^{*} P<0.05,{ }^{*} P<0.01$, and ${ }^{* * *} P<0.001$ versus VEH. LPS, lipopolysaccharide; poly(I:C), polyinosinic-polycytidylic acid; TLR, Toll-like receptor; VEH, vehicle.

trimester, LPS increased IL-8 mRNA $(P<0.01)$ and CCL2 mRNA levels $(P<0.05)$ after 4 and 24 hours $(P<0.05)$ (Figure 5, A and C). In the third trimester, LPS increased IL-8 mRNA levels after 4 hours $(P<0.05)$ and 24 hours $(P<0.01)$. CCL2 mRNA levels were increased $(P<0.05)$ after 4 hours of LPS exposure but returned to baseline after 24 hours (Figure 5, B and D).

Poly(I:C) induced significant increases in IL-8 $(P<0.01)$ and CCL2 $(P<0.01)$ mRNA levels after 24 hours in firsttrimester tissues (Figure 5, E and G). In third-trimester

tissues, poly(I:C) increased IL- 8 mRNA levels at 4 hours $(P<0.05)$ and 24 hours $(P<0.01)$, and CCL2 mRNA levels were increased after 4 hours $(P<0.05)$ and showed a strong trend toward increased CCL2 mRNA levels after 24 hours (Figure 5, F and $\mathrm{H}$ ).

\section{Villous Structural Integrity and Viability of First- and} Third-Trimester Explants

Placental explants before culture, after 24 hours of culture (equivalent to time 0 for LPS and poly(I:C) treatments), and after 48 hours of culture (equivalent to 24 hours of treatment) were examined to confirm the structural integrity of the explants (Figure 6A). NTRK2, the receptor for brain-derived neurotrophic factor, which is constitutively expressed in cytotrophoblast and syncytiotrophoblast in the human placenta ${ }^{26,27}$ clearly localized to those structures in the explants (Figure 6B). In addition, no effect of LPS or poly(I:C) was found on the release of LDH into the culture media in either first- or thirdtrimester explants (data not shown), indicating that explants were viable after culture and treatments.

\section{Discussion}

LPS (a component of Gram-negative bacterial wall) impaired placental mRNA and protein expressions of both transporters in the first-trimester explants but did not alter expression in the third trimester. In contrast, poly(I:C) (modeling viral infection) did not alter expression of the transporters in the first trimester but decreased the expression of ABCB1 mRNA in third-trimester explants after 24 hours of exposure. Expression of TLR-3 (the poly(I:C) receptor) and TLR-4 (the LPS receptor) increased from the first to the third trimester, and the localization of the receptors changed from cytotrophoblast early in gestation to syncytiotrophoblast at term. The expression of the receptors was not altered by LPS at either time in pregnancy. However, poly(I:C) decreased mRNA expression of both receptors in the third trimester but had no effect in the first trimester. In addition, we found a robust cytokine and chemokine response to LPS and poly(I:C) in both first- and third-trimester explants.

The multidrug transporters are a class of transporter proteins that provide a barrier function in many tissues (including the placenta) by inducing the efflux of drugs, toxins, and hormones from the intracellular space toward the extracellular space, or from one side of a barrier to the other. A number of studies in rodents have suggested that infection and inflammation can down-regulate several key drug transporters in the placenta. ${ }^{14}$ The extent to which bacterial- and viral-associated inflammation affect placental expression of $\mathrm{ABC}$ transporters in the human was largely unexplored.

In the mouse, previous reports documented that LPS impaired placental Abcbla mRNA expression. ${ }^{14}$ Acute sublethal LPS exposure decreased P-gp activity in the mouse placenta, ${ }^{12}$ promoting increased P-gp substrate accumulation in 
A

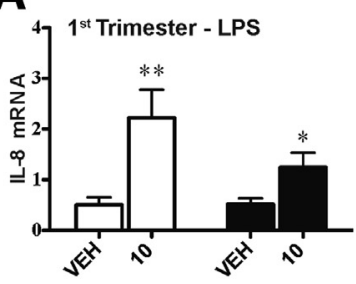

C

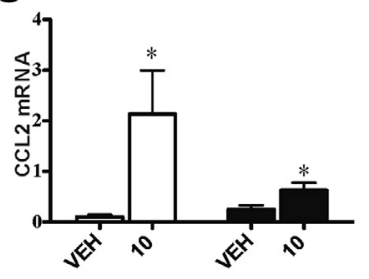

E
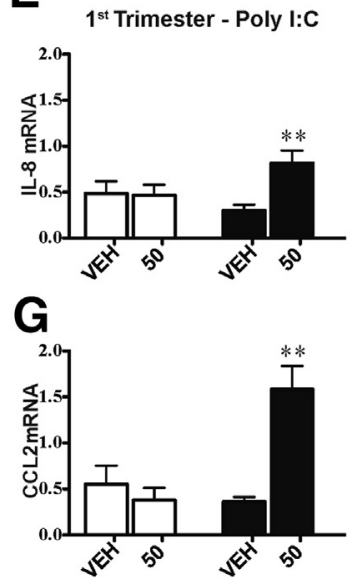

B

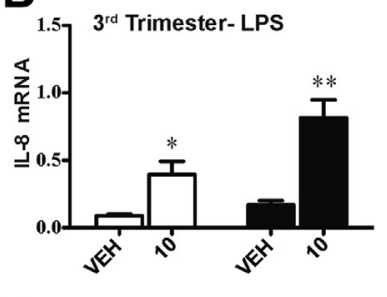

D

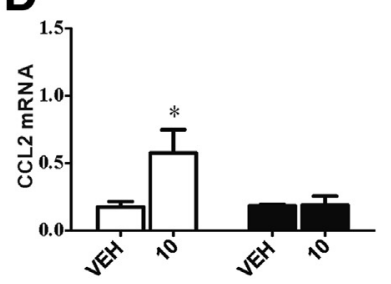

$\mathbf{F}$

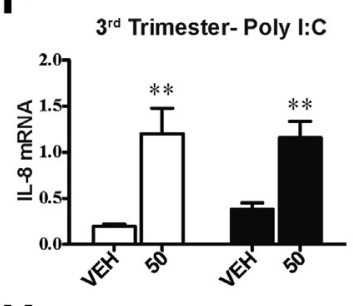

H

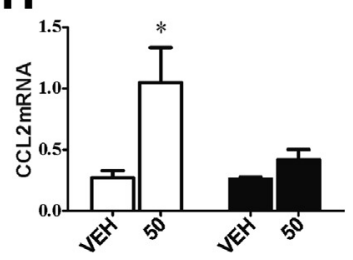

Figure 5 Effect of LPS and poly(I:C) on chemokine and cytokine mRNA expression in the first- and third-trimester placental villous explants. Differences in IL-8 (A) and CCL2 (C) mRNA levels between VEH and $10 \mu \mathrm{g} / \mathrm{mL}$ LPS treatment in the first trimester were assessed by paired $t$-test. In the third trimester the levels of IL-8 (B) and CCL2 (D) mRNA increase significantly (paired $t$-test) after exposure to $10 \mu \mathrm{g} / \mathrm{mL}$ LPS for 4 (white bars) or 24 (black bars) hours. Differences in IL-8 (E) and CCL2 (G) mRNA levels between VEH and $50 \mu \mathrm{g} / \mathrm{mL}$ poly(I:C) in the first trimester were assessed by paired $t$ test. In the third trimester the levels of IL-8 (F) and CCL2 (H) mRNA increase significantly (paired $t$-test) after exposure to $50 \mu \mathrm{g} / \mathrm{mL}$ poly(I:C) for 4 or 24 hours. Data are expressed as means \pm SEM. $n=6$ per group. ${ }^{*} P<0.05$, ${ }^{* * P}<0.01$ versus VEH. CCL2, chemokine (C-C motif) ligand 2; LPS, lipopolysaccharide; poly(I:C), polyinosinic-polycytidylic acid; $V E H$, vehicle.

the fetus. In the rat, LPS down-regulated placental Abcb1a/b and Abcg2 mRNA. ${ }^{15,28}$ Other studies found that LPS treatment decreased levels of Abcbla mRNA and protein in rat liver, brain, and heart. ${ }^{15,29}$ Together, these data suggest that infection and inflammation are capable of inducing changes in the levels of drug transporters, particularly P-gp and BCRP. This alters the biodistribution of xenobiotics in a variety of tissues and across biological barriers, including the placenta. This would result in increased fetal accumulation of toxins and xenobiotics that may be present at the maternal circulation. Our data also suggest that, at least with respect to drug transporters, the placenta exhibits a differential response to infectious agents and this effect is greater for bacterial challenge compared with that of viral

challenge. Moreover, the first-trimester placenta appears to be more sensitive to the effects of bacterial infection, potentially leading to increased exposure of the embryo or fetus to drugs and toxins at a critical time in development and that bacterial infection may have a greater impact on transporter expression in the first trimester.

Previous studies have found that poly(I:C) decreases the expression of placental Abcbla/b and Abcg 2 mRNA in the rat. ${ }^{16}$ In contrast, poly(I:C) induced the expression of Abcb1b mRNA but down-regulated P-gp and BCRP levels in rat liver. ${ }^{16}$ In the present study, the impact of viral and bacterial infection appears to be different, depending on gestational age. In the first trimester, explants were responsive to bacterial but not viral challenge. However, in the third trimester, expression of drug transporters is reduced by viral but not bacterial challenge. It is possible that a longer duration of exposure is required for an effect on drug transporter in third-trimester explants. Although 24 hours of exposure to infectious agents was sufficient to generate a response in the first trimester, this might not be the case for third-trimester explants, although we did find that first- and third-trimester explants mounted a similar inflammatory response (cytokines and chemokines) after LPS and poly(I:C) exposure. Further studies are required to determine the time dependency of these effects.

Developmental expression and localization of TLR-3 and TLR-4 may also play a role in determining the pattern of expression of drug transporters in response to bacterial and viral challenge. We found that TLR-4 is more abundantly expressed in the third-trimester placenta than in the first-trimester placenta, consistent with previous findings that TLR-4 expression is increased at term compared with earlier stages of pregnancy. ${ }^{17}$ In addition, we have shown, for the first time, that there is a gestational age-dependent pattern of expression for TLR-3, with increasing TLR-3 levels in the third-trimester placenta. This is somewhat counterintuitive, given that first-trimester placentas challenged with LPS showed decreased ABCB1 and ABCG2 expressions, whereas third-trimester placentas did not. These differences might be explained by the gestational age-dependent pattern of TLR-4 localization in the placenta. TLR-4 localized to the cytotrophoblast in the first trimester and in the syncytiotrophoblast layer in the thirdtrimester placenta as described previously. ${ }^{17}$ It may be that TLR-4 localized to the cytotrophoblast cells, when activated by TLR-4 ligands is capable of inducing changes in multidrug transporters in the first-trimester placenta, whereas activation of TLR-4 in the third-trimester syncytiotrophoblast is not.

Other than playing an important role in transporting xenobiotics, the $\mathrm{ABC}$ transporters also efflux a wide variety of substrates across cellular membranes, including metabolic products (amino acids), lipids (cholesterol), steroid hormones (ie, betamethasone), environmental toxins (ie, ivermectin and bisphenol-A), compounds involved in inflammatory response, and regulators of leukocyte invasion in non-uterine tissues. ${ }^{3,30}$ Importantly, blocking activity of P-gp in astrocytes reduced 
A
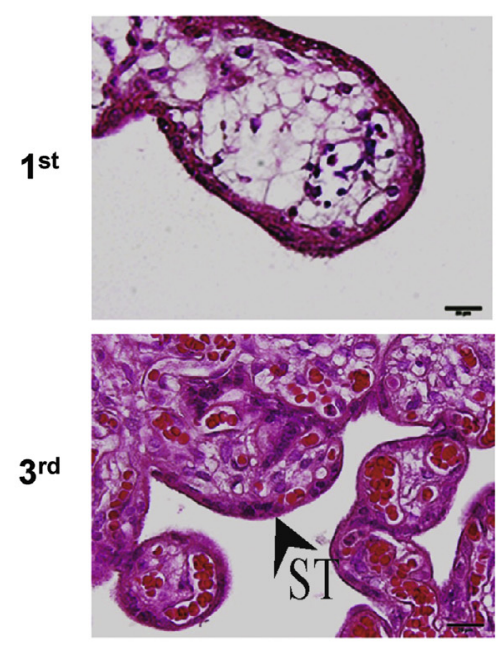

B

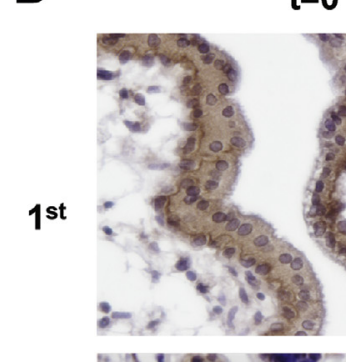

$t=0$

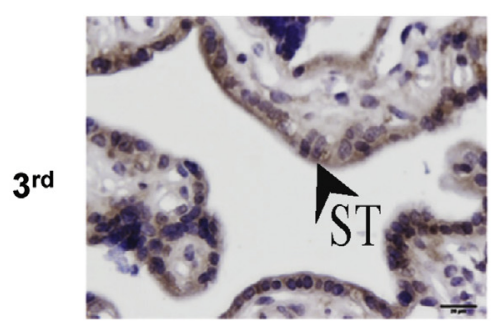

24h
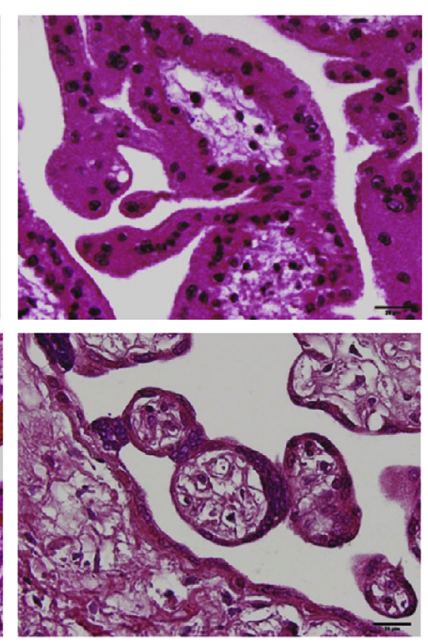

24h
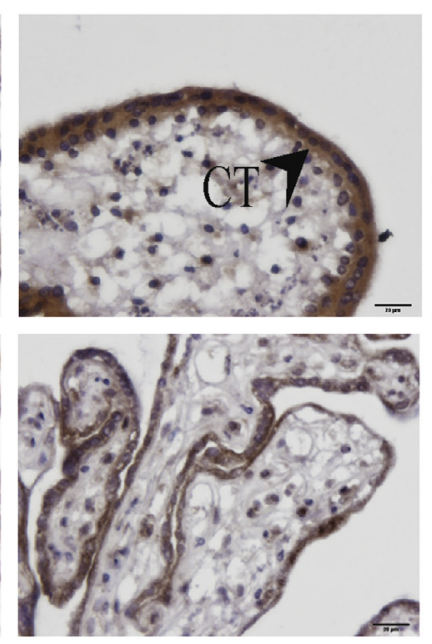

48h
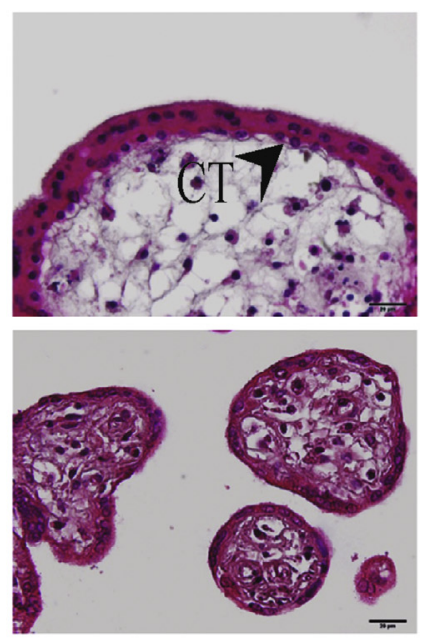

48h

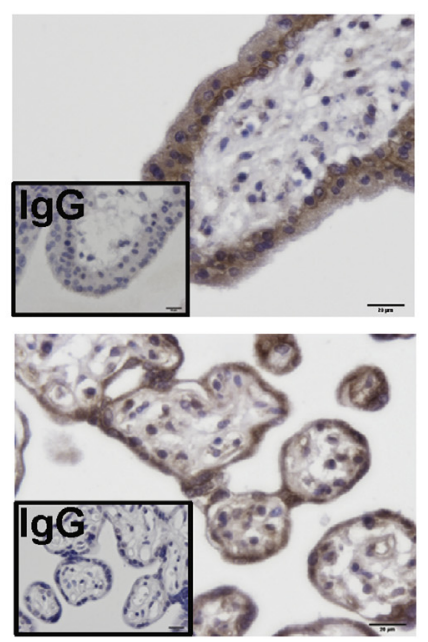

Figure 6 Immunohistochemical analysis shows explant integrity of first- and third-trimester explants. A: Representative histologic images of freshly dissected first- and third-villous explants $(t=0)$ and explants cultured for 24 and 48 hours at $8 \% 0_{2}$. B: Immunohistochemical staining of histologic sections shows ST and CT (identified by positive staining for NTRK2) integrity of freshly dissected first- and third-villous explants $(t=0)$ and explants cultured for 24 and 48 hours at $8 \% \mathrm{O}_{2}$. Insets: Mouse IgG1 was added instead of primary antibody in controls. Arrow represents CT and ST. $n=6$ per group. Scale bars: $20 \mu \mathrm{m}$ (A and B). CT, cytotrophoblast; ST, syncytiotrophoblast.

CCL2 secretion, ${ }^{31}$ an important uterine chemokine. ${ }^{32}$ Moreover, P-gp was shown to actively transport proinflammatory cytokines out of cells. ${ }^{31,33}$ As such, P-gp likely plays an important role in the secretion of proinflammatory and chemotactic factors into the extracellular space and therefore in the regulation of immunologic responses. In addition, the trophoblast can secrete a wide variety of cytokines and chemokines that are involved with immune chemotaxis, ${ }^{21,22,34,35}$ playing an important role in mediating local immunologic responses to protect the fetus from viral or bacterial infections. Therefore, it is tempting to speculate that modulation of the expression of multidrug transporters in response to bacterial and viral infections may also be part of the trophoblast-specific immunologic responses to infection, which seems to be gestational age and infective agent dependent.
It is also important to stress that TLRs are essential components of the signaling network within the innate immune response, which increase cytokine release to protect the fetus against preterm delivery in the context of infection. ${ }^{36,37}$ Our results indicate that LPS and poly(I:C) can elicit a robust cytokine and chemokine response (IL-8, CCL2) in both firstand third-trimester placental explants. Thus, the lack of effect of LPS or poly(I:C) on drug transporters in the third trimester is not due to a hyporesponsiveness to infection. Our results corroborate those of previous studies, indicating that the responsiveness of drug transporters to TLR ligands in trophoblast is highly gestational age dependant. ${ }^{12}$ We also found that LPS and poly(I:C) treatment did not alter explant viability (as determined by LDH measurements) or structure, suggesting that decreases in $\mathrm{ABCB} 1$ and $\mathrm{ABCG} 2$ expression are not likely caused by impaired explant viability. 


\section{Conclusion}

Our data show that bacterial and viral challenges can reduce the expression of the multidrug transporters in the human placenta. The modulation of these drug transporters is gestational age dependent, with the placenta being more sensitive to bacterial challenge during the first trimester and viral challenge in the third trimester. This does not appear to be due to a lack of response of recognition of these agents through TLRs, because activation of both TLR-3 and TLR-4 elicits robust cytokine responses in both the first and third trimesters. Because intrauterine infection and inflammation are relatively common during pregnancy (and associated with significant pregnancy disorders), the consequent reduction in the expression of drug transporters may expose the embryo or fetus to potentially harmful agents drugs, toxins, and hormones that cross from the maternal circulation at a time when it is most vulnerable.

\section{Acknowledgments}

P.L., E.B., W.G., S.J.L., and S.G.M. conceived and designed the experiments; P.L. and M.J. performed the experiments; P.L. analyzed the data; W.G., S.J.L., and S.G.M. contributed reagents, materials, and analysis tools; P.L., E.B., W.G., S.J.L., and S.G.M. wrote the paper.

\section{References}

1. Lecoeur S, Videmann B, Mazallon M: Effect of organophosphate pesticide diazinon on expression and activity of intestinal P-glycoprotein. Toxicol Lett 2006, 161:200-209

2. Mazur CS, Marchitti SA, Dimova M, Kenneke JF, Lumen A, Fisher J: Human and rat $\mathrm{ABC}$ transporter efflux of bisphenol a and bisphenol a glucuronide: interspecies comparison and implications for pharmacokinetic assessment. Toxicol Sci 2012, 128:317-325

3. Iqbal M, Audette MC, Petropoulos S, Gibb W, Matthews SG: Placental drug transporters and their role in fetal protection. Placenta 2012, 33: $137-142$

4. Lye P, Bloise E, Dunk C, Javam M, Gibb W, Lye SJ, Matthews SG: Effect of oxygen on multidrug resistance in the first trimester human placenta. Placenta 2013, 34:817-823

5. Sun M, Kingdom J, Baczyk D, Lye SJ, Matthews SG, Gibb W: Expression of the multidrug resistance P-glycoprotein, (ABCB1 glycoprotein) in the human placenta decreases with advancing gestation. Placenta 2006, 27:602-609

6. Yeboah D, Sun M, Kingdom J, Baczyk D, Lye SJ, Matthews SG, Gibb W: Expression of breast cancer resistance protein (BCRP/ABCG2) in human placenta throughout gestation and at term before and after labor. Can J Physiol Pharmacol 2006, 84: $1251-1258$

7. Behravan J, Piquette-Miller M: Drug transport across the placenta, role of the ABC drug efflux transporters. Expert Opin Drug Metab Toxicol 2007, 3:819-830

8. Petropoulos S, Gibb W, Matthews SG: Effect of glucocorticoids on regulation of placental multidrug resistance phosphoglycoprotein (P-gp) in the mouse. Placenta 2010, 31:803-810

9. Micuda S, Fuksa L, Mundlova L, Osterreicher J, Mokry J, Cermanova J, Brcakova E, Staud F, Pokorna P, Martinkova J: Morphological and functional changes in p-glycoprotein during dexamethasone-induced hepatomegaly. Clin Exp Pharmacol Physiol 2007, 34:296-303

10. Narang VS, Fraga C, Kumar N, Shen J, Throm S, Stewart CF, Waters CM: Dexamethasone increases expression and activity of multidrug resistance transporters at the rat blood-brain barrier. Am J Physiol Cell Physiol 2008, 295:C440-C450

11. Javam M, Audette MC, Iqbal M, Bloise E, Gibb W, Matthews SG: Effect of oxygen on multidrug resistance in term human placenta. Placenta 2014, 35:324-330

12. Bloise E, Bhuiyan M, Audette MC, Petropoulos S, Javam M, Gibb W, Matthews SG: Prenatal endotoxemia and placental drug transport in the mouse: placental size-specific effects. PLoS One 2013, 8:e65728

13. Dammann O, Leviton A: Role of the fetus in perinatal infection and neonatal brain damage. Curr Opin Pediatr 2000, 12:99-104

14. Chen YH, Wang JP, Wang H, Sun MF, Wei LZ, Wei W, Xu DX: Lipopolysaccharide treatment downregulates the expression of the pregnane $\mathrm{X}$ receptor, cyp3a11 and mdrla genes in mouse placenta. Toxicology 2005, 211:242-252

15. Wang JH, Scollard DA, Teng S, Reilly RM, Piquette-Miller M: Detection of P-glycoprotein activity in endotoxemic rats by $99 \mathrm{mTc}$ sestamibi imaging. J Nucl Med 2005, 46:1537-1545

16. Petrovic V, Piquette-Miller M: Impact of polyinosinic/polycytidylic acid on placental and hepatobiliary drug transporters in pregnant rats. Drug Metab Dispos 2010, 38:1760-1766

17. Beijar EC, Mallard C, Powell TL: Expression and subcellular localization of TLR-4 in term and first trimester human placenta. Placenta 2006, 27:322-326

18. Abrahams VM, Visintin I, Aldo PB, Guller S, Romero R, Mor G: A role for TLRs in the regulation of immune cell migration by first trimester trophoblast cells. J Immunol 2005, 175:8096-8104

19. Patni S, Wynen LP, Seager AL, Morgan G, White JO, Thornton CA: Expression and activity of Toll-like receptors 1-9 in the human term placenta and changes associated with labor at term. Biol Reprod 2009, $80: 243-248$

20. Torricelli M, Novembri R, Bloise E, De Bonis M, Challis JR, Petraglia F: Changes in placental CRH, urocortins, and CRH-receptor mRNA expression associated with preterm delivery and chorioamnionitis. J Clin Endocrinol Metab 2011, 96:534-540

21. Novembri R, Torricelli M, Bloise E, Conti N, Galeazzi LR, Severi FM, Petraglia F: Effects of urocortin 2 and urocortin 3 on IL-10 and TNFalpha expression and secretion from human trophoblast explants. Placenta 2011, 32:969-974

22. Torricelli M, Voltolini C, Bloise E, Biliotti G, Giovannelli A, De Bonis M, Imperatore A, Petraglia F: Urocortin increases IL-4 and IL10 secretion and reverses LPS-induced TNF-alpha release from human trophoblast primary cells. Am J Reprod Immunol 2009, 62: 224-231

23. Anton L, Brown AG, Parry S, Elovitz MA: Lipopolysaccharide induces cytokine production and decreases extravillous trophoblast invasion through a mitogen-activated protein kinase-mediated pathway: possible mechanisms of first trimester placental dysfunction. Hum Reprod 2012, 27:61-72

24. Kayaalti Z, Tekin D, Aliyev V, Yalcin S, Kurtay G, Soylemezoglu T: Effects of the interleukin-6 (IL-6) polymorphism on toxic metal and trace element levels in placental tissues. Sci Total Environ 2011, 409: 4929-4933

25. Drewlo S, Levytska K, Kingdom J: Revisiting the housekeeping genes of human placental development and insufficiency syndromes. Placenta 2012, 33:952-954

26. Kawamura K, Kawamura N, Kumazawa Y, Kumagai J, Fujimoto T, Tanaka T: Brain-derived neurotrophic factor/tyrosine kinase B signaling regulates human trophoblast growth in an in vivo animal model of ectopic pregnancy. Endocrinology 2011, 152:1090-1100

27. Dunk CE, Roggensack AM, Cox B, Perkins JE, Åsenius F, Keating S, Weksberg R, Kingdom JCP, Adamson SL: A distinct microvascular endothelial gene expression profile in severe IUGR placentas. Placenta 2012, 33:285-293 
28. Petrovic V, Wang JH, Piquette-Miller M: Effect of endotoxin on the expression of placental drug transporters and glyburide disposition in pregnant rats. Drug Metab Dispos 2008, 36:1944-1950

29. Piquette-Miller M, Pak A, Kim H, Anari R, Shahzamani A: Decreased expression and activity of P-glycoprotein in rat liver during acute inflammation. Pharm Res 1998, 15:706-711

30. van de Ven R, Oerlemans R, van der Heijden JW, Scheffer GL, de Gruijl TD, Jansen G, Scheper RJ: ABC drug transporters and immunity: novel therapeutic targets in autoimmunity and cancer. J Leukoc Biol 2009, 86:1075-1087

31. Kooij G, Mizee MR, van Horssen J, Reijerkerk A, Witte ME, Drexhage JA, van der Pol SM, van Het Hof B, Scheffer G, Scheper R, Dijkstra CD, van der Valk P, de Vries HE: Adenosine triphosphatebinding cassette transporters mediate chemokine (C-C motif) ligand 2 secretion from reactive astrocytes: relevance to multiple sclerosis pathogenesis. Brain 2011, 134:555-570

32. Shynlova O, Tsui P, Dorogin A, Lye SJ: Monocyte chemoattractant protein-1 (CCL-2) integrates mechanical and endocrine signals that mediate term and preterm labor. J Immunol 2008, 181: $1470-1479$
33. Pawlik A, Baskiewicz-Masiuk M, Machalinski B, Safranow K, Gawronska-Szklarz B: Involvement of P-glycoprotein in the release of cytokines from peripheral blood mononuclear cells treated with methotrexate and dexamethasone. J Pharm Pharmacol 2005, 57: $1421-1425$

34. Bloise E, Torricelli M, Novembri R, Borges LE, Carrarelli P, Reis FM, Petraglia F: Heat-killed Lactobacillus rhamnosus GG modulates urocortin and cytokine release in primary trophoblast cells. Placenta 2010, 31:867-872

35. Dowling O, Chatterjee PK, Gupta M, Tam Tam HB, Xue X, Lewis D, Rochelson B, Metz CN: Magnesium sulfate reduces bacterial LPSinduced inflammation at the maternal-fetal interface. Placenta 2012, 33:392-398

36. Romero R, Gotsch F, Pineles B, Kusanovic JP: Inflammation in pregnancy: its roles in reproductive physiology, obstetrical complications, and fetal injury. Nutr Rev 2007, 65:S194-S202

37. Kumazaki K, Nakayama M, Yanagihara I, Suehara N, Wada Y: Immunohistochemical distribution of Toll-like receptor 4 in term and preterm human placentas from normal and complicated pregnancy including chorioamnionitis. Hum Pathol 2004, 35:47-54 